\title{
Dynamic Channel Allocation and Optimal Detection for MAC in CDMA Ad hoc Networks
}

\author{
Amit Butala and Lang Tong
}

\begin{abstract}
A medium-access control (MAC) protocol for spread-spectrum ad-hoc networks with Dynamic Channel Allocation (DCA) is presented. DCA can support large systems with smaller spreading gains by dynamically assigning channels only when a node has a packet to transmit. It is shown that DCA is collision free. By assigning channels dynamically, DCA offers improved throughput normalized by available bandwidth.
\end{abstract}

\section{INTRODUCTION}

One of the challenges in the design of multihop ad hoc network is medium access control (MAC). When there is a single channel, an effective class of MAC protocols are based on a combination of carrier sensing, control packets (RTS-CTS), and busy tones. In such protocols as MACAW [3], DBTMA [4], and FAMA [5], a node may transmit only if the control packet carrying the request for transmission is acknowledged by the intended receiver. The dynamic scheduling (in time) enables an efficient utilization of channel resources.

MAC protocols employing spread-spectrum signaling have also garnered considerable attention [6], [7], [8]. For spread spectrum ad hoc networks, it is possible (and often desirable) that multiple spreading codes are used. This adds the code dimension to the medium access control problem where multiple channels can be allocated to different users. If the spreading sequences have good crosscorrelation properties, contention on one channel does not affect transmission on the other channels. Thus, a protocol can be set up in which the exposed nodes and the hidden nodes may also transmit without destroying ongoing data transmissions.

For spread-spectrum ad hoc networks with multiple channels, the issue of efficiency of spectral utilization arises. Because the number of available channels in spread spectrum networks is related to the available bandwidth, one needs to allocate channels efficiently in the sense that the throughput normalized by the bandwidth is maximized. While the number of codes to be used in a network can be predetermined, the number of potential users is often unknown. This makes it necessary to have a MAC scheme that allocates channels in a dynamic fashion, and at the same time, avoids problems associated with hidden/exposed terminals. Unfortunately, spread-spectrum

Amit Butala is with Qualcomm Inc., abutala@qualcomm.com.

Lang Tong is with the School of Electrical and Computer Engineering, 384 Rhodes Hall, Cornell University, Ithaca, NY 14853. lt ong@ee. cornell.edu.

This work was supported in part by the Multidisciplinary University Research Initiative (MURI) under the Office of Naval Research Contract N00014-00-1-0564. schemes designed so far increase the number of channels required in accordance with either the size of the network [6], [7], or the length of the data-packet [8].

In this paper, we propose a new MAC protocol for multihop spread-spectrum ad hoc networks. For a fixed number of channel (spreading codes), the MAC protocol, referred to as Dynamic Channel Allocation (DCA), allocates channels on-demand. Using a combination of RTS-CTS messaging, a query packet, and a busy tone, DCA is a collision-free MAC protocol with higher normalized throughput than that of existing techniques. Without assuming perfect orthogonality of codes, DCA is also the first MAC protocol where the channel assignment is done on the basis of the interference modeled at the receiver. We show that this scheme of channel allocation with interference detection ensures the perfect reception of data-packets under ideal conditions.

\section{Network Model and Performance Measure}

\section{A. The Model}

We use the protocol model definition for the neighborhood of a node. Thus, each node within a fixed radius $(R)$ of the transmitter is assumed to be contained in its neighborhood and can listen to the transmitter. The relationship is dual: a node is not affected by any transmission that originates outside its neighborhood. The assumption of fixed transmission power is implicit to the model. The coverage areas for the nodes are represented by the circles centered at the respective nodes.

We assume $M+1$ distinct spreading codes available for transmission, one of which is reserved for transmission of control sequences and the rest for data. The ad hoc network consists of $N$ nodes where $N$ can be much larger than $M+1$. Nodes are half duplex and can tune to only one channel at any given time. In addition, nodes also have a frequency generator/receiver that may be used to transmit/receive a monotone on a preset frequency. The monotone is used to specify a busy signal during packet reception. Issues related to routing are not considered. It is assumed that the nodes either know the routing tables a priori or the range of communication involves only neighboring nodes.

Transmission time is slotted, and the transmissions are packet synchronized. The data is broken up into minipackets that are transmitted in succession, with each minipacket requiring one time-slot. The RTS and the CTS packets are assumed to be less than one half mini-packet in length such that a RTS-CTS packet exchange between any two nodes in the network may be completed in a single 
mini-slot.

\section{B. A Performance Measure: Normalized Throughput}

Since the number of codes in the system that satisfy the constraints on multi-access interference is proportional to the spreading gain, the absolute performance cannot be inferred simply by observing the raw network throughput. The network throughput is expected to increase with an increase of spreading gain and hence we introduce the concept of normalized throughput for comparison of different protocols.

The network throughput $(\Gamma)$ is defined as the average number of packets successfully received in one time-slot over the network when in steady state. The spreading gain $(G)$ is the ratio of the chip rate to the symbol rate of a spread-spectrum signal. Then the normalized throughput $(\eta)$ can be defined as the ratio of the network throughput to the spreading gain,

$$
\eta=\frac{\Gamma}{G}
$$

For simplicity, in all the subsequent discussions the spreading gain is taken to be equal to the total number of codes available to the system and the protocol efficiencies are compared against their normalized throughput.

\section{Dynamic Channel Allocation}

Our objective is to develop a protocol that can optimize the throughput between the nodes in the network. The operational issues are the scheduling of transmissions and the assignment of channels to the nodes. A demand-driven dynamic allocation of channels is proposed.

Dynamic Channel Allocation relies on the assignment of one of the available data-channel to the nodes that get scheduled to transmit. Thus, the two basic requirements for packet exchange are,

Scheduling: For a successful transmission, there should be only one transmitter attempting to transmit to a node, and any such transmission must be destined to an idle node. This is effected by the transmission of the RTS-CTS on the control channel. Since the channel is a collision channel and multiple transmissions on the same channel result in packet collision, the RTS-CTS ensures proper scheduling of the transmissions.

Allocation: Given that two terminals are scheduled, there must be a channel available for transmission that does not interfere with any on-going transmission. This is effected by a new procedure called Querying of channels.

\section{A. Querying of Channels}

The RTS-CTS control packet exchange establishes the scheduling of packets over a particular channel, but cannot ascertain the availability of the channel. A channel is said to be available only if no node in the neighborhood of the intended receiver is transmitting on that channel, and no other node in the neighborhood of the intended transmitter is receiving on that channel. These are, respectively, the conventional exposed terminal and hidden terminal problems in the multi-channel scenario.

The solution proposed here is the transmission of the Query by any node that intends to transmit a data packet. The Query is a known data-packet and thus is a deterministic interference that may be estimated. Once a datatransmission is scheduled using the RTS-CTS exchange, the transmitter sends out the Query on the selected channel. In response to contention caused by the Query, if any, any receiver may transmit a busy tone. The busy tone is a sinusoid sent on an out-of-band frequency, and intimates the transmitter that the channel is in use. A Query is successful only if no busy tone is heard by the transmitter. This represents the case that no exposed terminal is receiving and no hidden terminal is transmitting on the selected channel. A node may transmit only if its Query is successful.

With the introduction of the Query, in each time-slot, all the nodes may be classified into the following 4 states:

- Idle (or Backlogged) state: Nodes that are not engaged in packet reception or transmission.

- Query state: Nodes that get scheduled and are transmitting the Query in the current time-slot.

- Data state: Nodes involved in transmission or reception of data packets. Only nodes in the Data state successfully transmit data over the network.

- Locked state: An extra state that tracks nodes involved in data-packet collisions. This occurs due to a mis-detection of the Query and shall be been discussed in greater detail in the next section.

\section{B. The Protocol}

The Dynamic Channel Allocation protocol is defined below and has been illustrated in Fig. 1, Fig. 2 and Fig. 3.

- Any idle node (e.g. A) that has a packet to transmit to any of its immediate neighbors (e.g. B) attempts to establish a communication by broadcasting an RTS on the common channel at the beginning of the mini-slot (Fig. 2 and Fig. 3).

- The RTS contains the following information: the destination node (B) identifier, the transmitting node (A) identifier, and the selected channel $(\mathbf{Q})$ on which the data will follow. The channel ( $\mathbf{Q})$ may be randomly chosen from the set of available channels.

- If the destination node, B, receives the RTS, it responds immediately, in the same time-slot, with a CTS on the common channel (Fig. 2 and Fig. 3). B transitions from the Idle state to the Query state for the next time-slot and tunes its receiver to the selected channel, $\mathbf{Q}$.

- If A does not receive a CTS in the same time-slot, it times out and reverts back to the Idle state. A re-transmission is attempted according to the back-off strategy. If $\mathrm{A}$ receives a CTS, it moves from the Idle state to the Query state. This completes the scheduling.

- In the next mini-slot, A transmits a Query on the selected channel. The Query is successful if no busy tone is generated (Fig. 2).

- The busy tone is generated in two possible cases: 
- By the intended receiver, $\mathrm{B}$, if the Queried channel is already in use (Fig. 1.b)

- By the contended receiver, $\mathrm{D}$, if the selected channel is already in use (Fig. 1.c)

- If $\mathrm{A}$ receives a busy tone on the busy-tone frequency, (cases b or c) it aborts transmission on the channel and reverts to the Idle state (Fig. 3). If $\mathrm{A}$ does not receive a busy tone on the busy-tone frequency, it moves to the Data state and begins transmission of the data packet from the time-slot that follows (Fig. 2).

- At the end of the data transmission interval, which is an integral number of mini-slots, both $\mathrm{A}$ and $\mathrm{B}$ reset to the Idle state.

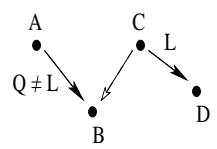

(a)

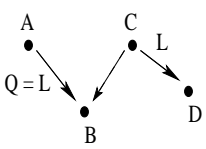

(b)

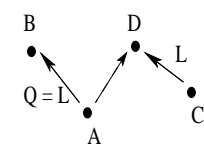

(c)
Fig. 1. Query for different network states: a) success b) failure c) failure

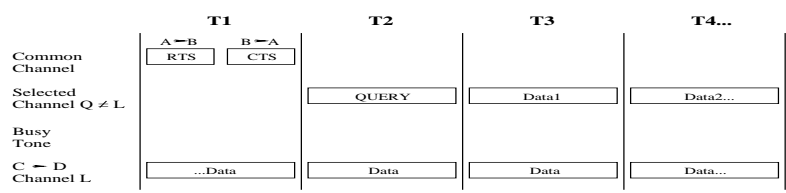

Fig. 2. Successful Querying: case (a)

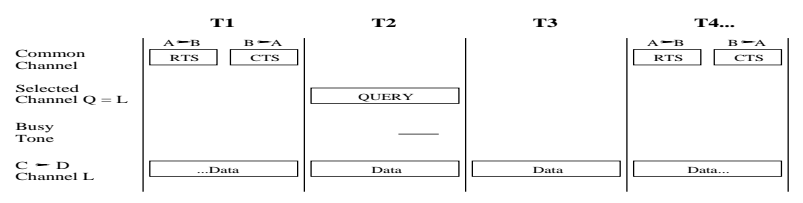

Fig. 3. Failed Querying: cases (b) and (c)

Property 1: Under the assumption of perfect detection of the Query, there are no data-packet collisions.

\section{Optimal Detection of Query}

In the presence of noise and multi-access interference, the detection of the Query is not perfect and is contingent on the operating characteristics of the receivers. At every receiver, the interference due to the Query may be missed or a false alarm be raised in response to a Query that does not interfere. This results in a probabilistic model for the acceptance of the Query.

Missed-detection: In the case of a Missed-detection, there are two nodes transmitting on the same channel within the vicinity of the receiver. This results in a packet collision at the receiver and it is unable to detect either packet. The receiver and the corresponding transmitted are assumed to be in the Locked state. The throughput of node-pairs in the Locked state is zero.

Transition of node-pairs from the Locked state to the Data state would depend on the coding scheme used and the higher layers. Without imposing any additional constraints, we assume that the pair remains in the Locked state till the end of the current data-packet transmission, after which they reset to the Idle state.

False-alarm: The False-alarm is less damaging, since it merely results in the node $\mathbf{A}$ aborting the transmission of the data-packet and reverting back to the Idle state. A retransmission is attempted in accordance with the protocol. This too, would lead to a decrease in the throughput of the network.

The two events are inversely proportional to each other, thus the optimization of the throughput requires an analysis of the receiver operating characteristics. We've shown that [13], under the knowledge of the Signal-to-Noise ratio, the detection of the Query can be modeled as a NeymanPearson detector.

For an $\alpha$ level receiver (i.e. probability of False-alarm $P_{\alpha}=\alpha$ ), the power of the detector, at a signal-to-noise ratio of $S N R$ is given by,

$$
P_{D}=2 \mathbf{Q}\left[\frac{\mathbf{Q}^{-1}\left(\frac{P_{\alpha}}{2}\right)}{\sqrt{\kappa S N R+1}}\right] .
$$

\section{Results and Simulations}

The maximum throughput of DCA is prone to the operating characteristics of the detector for the Query. Peak throughput depends on both the probability of False-alarm as well as Missed-detection. Each receiver may pick up its operating point based on it's individual requirements. For simplicity, however, we assume that all receivers operate at the same point on the ROC. The throughput then relates to the ROC as shown earlier in figure 4 . Once the system SNR is computed at the receiver, the threshold of the detector is set at the point on the ROC curve that maximizes the throughput.

A comparison of three schemes, MACA-CT, CHMA, and DCA, is made for a fully connected 20 node network carrying data-packets geometrically distributed in length and with a mean length of 10 mini-packets. The number of data-channels depends on the protocol. For DCA, we randomly choose 5 data-channels. MACA-CT has 20 channels, determined by the size of the network. For CHMA, this number would have to be greater than the largest data-packet in the network, which is infinity. We compare against the normalized throughput of Modified-CT which illustrates the best case performance of CHMA for a channel hopping sequence that is twice as long as the length of the average data-packet length [13].

The normalized throughput of the 3 protocols are plotted in figure 5. The Query detector for DCA is assumed to be operating at $2 \mathrm{~dB}$ SNR. 


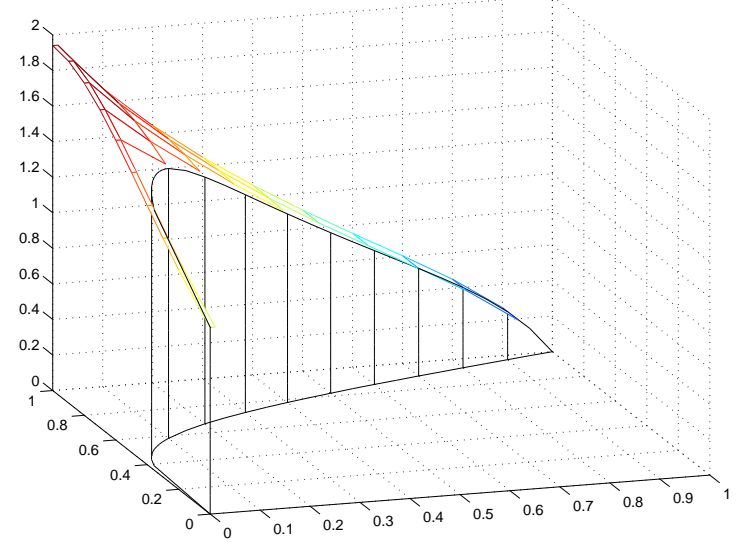

Fig. 4. Throughput of DCA for a fully connected network with 10 nodes, 5 data-channels and a mean data-packet length of 10 minipackets for different values of the threshold at $2 \mathrm{~dB}$.

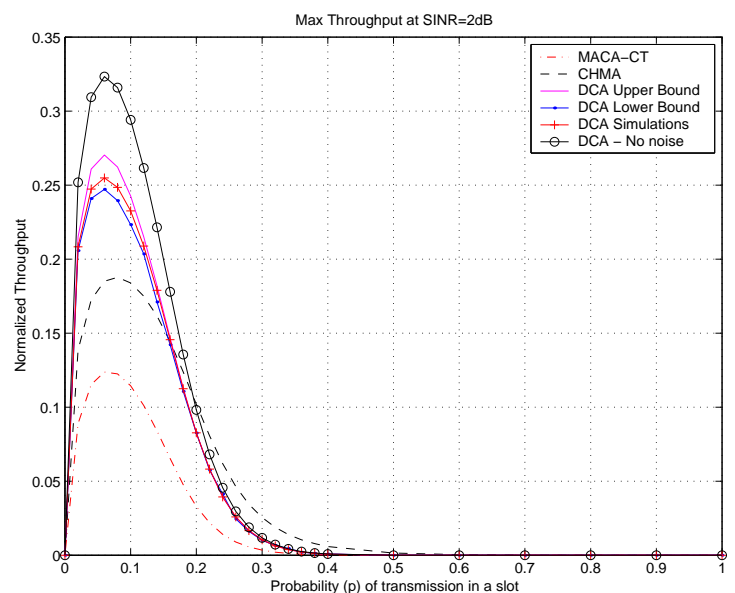

Fig. 5. Normalized throughput for MACA-CT, CHMA, DCA at 2dB, and DCA in the persence of no noise

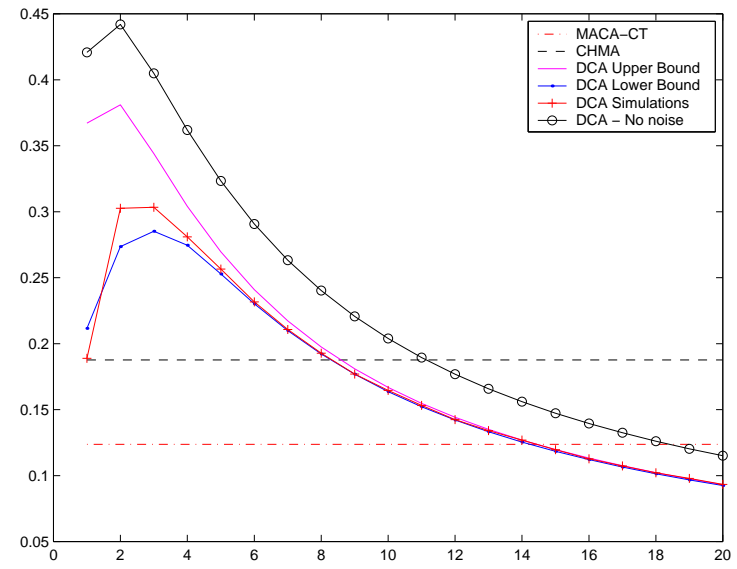

Fig. 6. Throughput as a function of the number of channels for a 20 Node network with average data-packet length of 10 mini-packets
Also, from figure 6, the normalized throughput appears to monotonically decreasing beyond the addition of the first few codes. The best case performance is for systems with 2 to 4 data-channels. The results are not totally surprising since one might expect the control channel to be the bottle neck as more channels are made available for Data. Increasing the available number of channels does not yield to a proportionate increase in data traffic. Interestingly, performance of DCA is superior till the number of channels equals 8. Fixed channel allocation schemes would yield better throughput than DCA if more channels might be made available.

\section{A. Multi-hop Networks}

All the above analysis are for a fully connected single hop scenario. Modeling of a multi-hop network is difficult. However, our simulations confirm the applicability of the protocol to multi-hop networks and its superiority over existing protocols for the few different topologies tested.

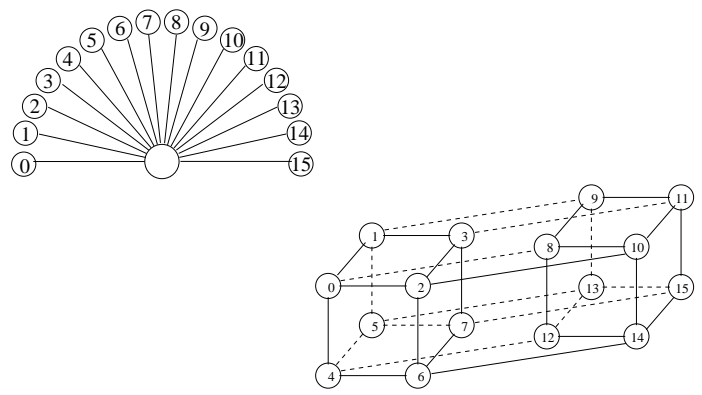

Fig. 7. 16 node ad hoc networks

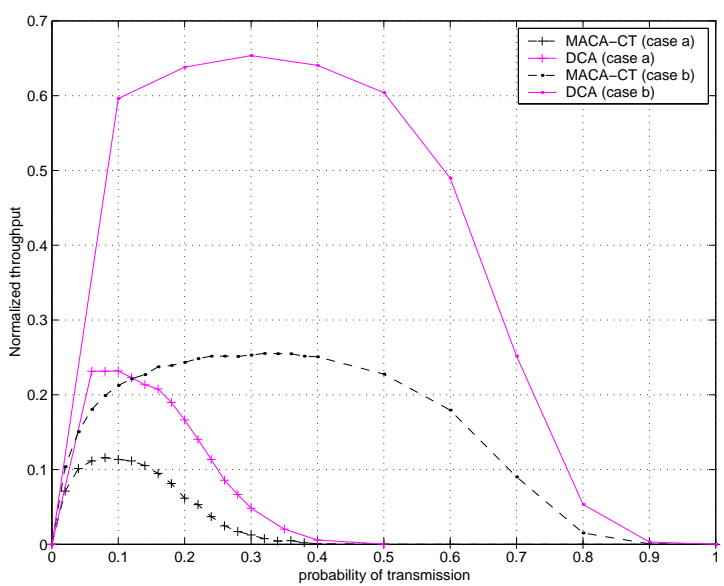

Fig. 8. Throughput comparisons for different scenarios

Figure 7.a shows a fully-connected network in which all the traffic is directed to the base station. Figure 7.b is a multi-hop network of 16 nodes with each node having 4 neighbors. The lines between the nodes show the connectivity between the nodes.

It is interesting to note the structural dependence on the requirement of the number of spreading codes for the other 
protocols. In case b, MACA-CT can be designed using a minimal of 11 data codes by taking advantage of spatial separation. For either situation, CHMA would still require as many channels as the maximal data-packet length. Both problems can be avoided by a dynamic allocation of channels.

The parameters used in the simulations are identical to those used previously. We consider 5 data-channels with one common control channel. Mean data-packet length is 10 slots, with a geometric distribution. Nodes have a single packet buffer. The network throughput is recorded with a constant probability of packet arrival $(p)$.

As can be seen, for case b, since the contention neighborhood is much smaller, the throughput of DCA is significantly greater that that for a fully connected network of the same size. Also the gains of DCA over MACA-CT are clearly visible.

Thus, in the context specified, DCA is superior to the other protocols and offers significant advantage. The penalty is the increased complexity of the receiver and the need for proper parameter selection. These could either be set apriori or kept variant, dependent on the network load.

\section{Conclusions}

Medium access control is a critical issue in ad hoc networks. One of the biggest stumbling block remains the proper scheduling of hidden and exposed terminals in the absence of a centralized contention resolution scheme. Contention of data-packets occurs at the receivers and hence, proper scheduling of data-packets requires the propogation of the contention information from the receivers to the transmitters. This is particularly interesting for multichannel ad hoc networks since the contention information can also be used in channel allocation.

In multi-channel ad hoc networks, the channel assignment has conventionally been regarded as a separate issue and isolated from the MAC. The spreading gain and consequent loss in the data-rate are mostly overlooked.

Our objective here has been to propose a MAC protocol for multichannel ad hoc networks based on the feedback of channel contention at the receiver. A channel is selected for transmission only if it does not cause any contention at any of the receivers in the neighborhood. The protocol is proposed in Section III.

The salient features of the protocol include, introduction of an optimal detector and busy tone mechanism to propogate channel contention information to the transmitters in the network, and channel allocation as a part of the MAC. This results not only in a tighter reuse of channels over a multi-hop network but also makes the spreading gain independent of the size of the neighborhood.

The throughput of the protocol is presented in section IV. Our analysis and simulations reveal that the network throughput is a convex function of the spreading codes, data-packet length and the probability of transmission. The operating threshold of the Query detection also has significant impact on the network throughput. Proper selection of network parameters is crucial in order to maxi- mize the throughput.

Performance of the system for different parameters is analyzed in section V. It is seen that DCA is superior to other protocols. DCA also manages to reduce the dependence of the protocol on the network topology thus being more versatile.

\section{REFERENCES}

[1] L. Kleinrock and F.A. Tobagi - "Packet switching in radio channels: Parts I \& II , IEEE Trans. Communications, vol. COM-23, No. 12, pp. 1400-1433, Dec 1975.

[2] P. Karn, "MACA - a new channel access method for packet radio", in ARRL/CRRL Amateur Radio 9th Computer Networking Conference, pp. 134-40, ARRL, 1990.

[3] V. Bharghavan, A. Demers, S. Shenker, and L. Zhang, "MACAW: A Media Access Protocol for Wireless LANs", in Proc. ACM SIGCOMM 1994, pp. 212-225, Sept. 1994.

[4] Z.J. Haas, Jing Deng and S. Tabrizi, " Collision-free medium access control scheme for ad-hoc networks", in Proc. IEEE MILCOM 1999, Vol 1, pp. 276-280, 1999.

[5] C. L. Fullmer and J.J. Garcia-Luna-Aceves, "Floor Acquisition Multiple Access (FAMA) for Packet-Radio Networks", in Proc. ACM SIGCOMM 1995, Cambridge, MA, Sept. 1995.

[6] E.S. Sousa and J.A. Silvester, "Spreading code protocols for distributed spread-spectrum packet radio networks", IEEE Trans. Communications, vol 36, pp. 272-281, March 1988.

[7] M. Joa-Ng and I-Tai Lu, "Spread-spectrum medium access protocol with collision avoidance in mobile ad-hoc wireless network", in Proc. IEEE INFOCOM 1999, vol. 2, pp. 776-783, 1999.

[8] A. Tzamaloukas and J.J. Garcia-Luna-Aceves, "Channel-hopping multiple access", in Proc. IEEE ICC 2000, vol. 1, pp. 415-419, 2000.

[9] Sanjay Lal and E.S. Sousa, "Distributed resource allocation for DS-CDMA-based multimedia ad hoc wireless LANs", IEEE Journal on Selected Areas in Communications, vol. 17, pp. 947-967, May 1999.

[10] A. Tzamaloukas and J.J. Garcia-Luna-Aceves, "A receiverinitiated collision-avoidance protocol for multi-channel networks", in Proc. IEEE INFOCOM 2001, vol. 1, pp189-198, 2001.

[11] E.S. Sousa, "Interference modeling in a direct-sequence spreadspectrum packet radio network", IEEE Trans. Communications, Vol. 38, Issue 9, pp1475-1482, Sept. 1990.

[12] IEEE P802.11 Draft Standard for Wireless LAN: Medium Access Control (MAC) and Physical Layer (PHY) Specifications, IEEE, July 1996.

[13] Amit Butala, "Dynamic channel allocation for medium access control in spread-spectrum ad hoc networks", Master's Dissertation, Cornell University, August 2001. 